\title{
A Phase 3 Trial of Bevacizumab in Ovarian Cancer
}

\author{
Timothy J. Perren, M.D., Ann Marie Swart, M.D., Jacobus Pfisterer, M.D., \\ Jonathan A. Ledermann, M.D., Eric Pujade-Lauraine, M.D., Gunnar Kristensen, M.D., \\ Mark S. Carey, M.D., Philip Beale, M.D., Andrés Cervantes, M.D., \\ Christian Kurzeder, M.D., Andreas du Bois, M.D., Jalid Sehouli, M.D., \\ Rainer Kimmig, M.D., Anne Stähle, M.D., Fiona Collinson, M.D., \\ Sharadah Essapen, M.D., Charlie Gourley, M.D., Alain Lortholary, M.D., \\ Frédéric Selle, M.D., Mansoor R. Mirza, M.D., Arto Leminen, M.D., \\ Marie Plante, M.D., Dan Stark, M.D., Wendi Qian, Ph.D., Mahesh K.B. Parmar, Ph.D., \\ and Amit M. Oza, M.D., for the ICON7 Investigators*
}

\section{ABSTRACT}

\section{BACKGROUND}

The authors' affiliations are listed in the Appendix. Address reprint requests to Dr. Parmar at MRC CTU, Aviation House, 125 Kingsway, London WL2B 6NH, United Kingdom, or at mp@ctu.mrc.ac.uk.

*Additional investigators participating in the International Collaboration on Ovarian Neoplasms (ICON7) are listed in the Supplementary Appendix, available at NEJM.org.

This article (10.1056/NEJMoal103799) was updated on December 29, 2011, at NEJM .org.

N Engl J Med 2011;365:2484-96. Copyright @ 2011 Massachusetts Medical Society.
Angiogenesis plays a role in the biology of ovarian cancer. We examined the effect of bevacizumab, the vascular endothelial growth factor inhibitor, on survival in women with this disease.

METHODS

We randomly assigned women with ovarian cancer to carboplatin (area under the curve, 5 or 6 ) and paclitaxel (175 mg per square meter of body-surface area), given every 3 weeks for 6 cycles, or to this regimen plus bevacizumab ( $7.5 \mathrm{mg}$ per kilogram of body weight), given concurrently every 3 weeks for 5 or 6 cycles and continued for 12 additional cycles or until progression of disease. Outcome measures included progressionfree survival, first analyzed per protocol and then updated, and interim overall survival.

RESULTS

A total of 1528 women from 11 countries were randomly assigned to one of the two treatment regimens. Their median age was 57 years; $90 \%$ had epithelial ovarian cancer, $69 \%$ had a serous histologic type, $9 \%$ had high-risk early-stage disease, $30 \%$ were at high risk for progression, and 70\% had stage IIIC or IV ovarian cancer. Progressionfree survival (restricted mean) at 36 months was 20.3 months with standard therapy, as compared with 21.8 months with standard therapy plus bevacizumab (hazard ratio for progression or death with bevacizumab added, 0.81 ; $95 \%$ confidence interval, 0.70 to $0.94 ; \mathrm{P}=0.004$ by the log-rank test). Nonproportional hazards were detected (i.e., the treatment effect was not consistent over time on the hazard function scale) ( $\mathrm{P}<0.001)$, with a maximum effect at 12 months, coinciding with the end of planned bevacizumab treatment and diminishing by 24 months. Bevacizumab was associated with more toxic effects (most often hypertension of grade 2 or higher) $(18 \%$, vs. $2 \%$ with chemotherapy alone). In the updated analyses, progression-free survival (restricted mean) at 42 months was 22.4 months without bevacizumab versus 24.1 months with bevacizumab $(\mathrm{P}=0.04$ by log-rank test); in patients at high risk for progression, the benefit was greater with bevacizumab than without it, with progression-free survival (restricted mean) at 42 months of 14.5 months with standard therapy alone and 18.1 months with bevacizumab added, with respective median overall survival of 28.8 and 36.6 months.

CONCLUSIONS

Bevacizumab improved progression-free survival in women with ovarian cancer. The benefits with respect to both progression-free and overall survival were greater among those at high risk for disease progression. (Funded by Roche and others; ICON7 Controlled-Trials.com number, ISRCTN91273375.) 
VARIAN CANCER IS THE FOURTH MOST common cause of cancer-related deaths in women, with an estimated 200,000 cases and 125,000 deaths occurring annually worldwide. For the past decade, the standard treatment for women with advanced ovarian cancer has been surgery and platinum-based chemotherapy. Attempts to improve this standard two-drug chemotherapy by adding a third cytotoxic drug failed to affect either progression-free survival or overall survival and resulted in an increase in toxic effects. ${ }^{1-4}$ Although intraperitoneal chemotherapy has extended overall survival by 12 to 17 months, it is an option only for women with advanced ovarian cancer who have a small amount of residual disease after surgery ${ }^{5-7}$ and is not widely used. Since the results of one trial suggested that weekly paclitaxel administration provided some benefit, ${ }^{8}$ trials are now under way to optimize carboplatin and paclitaxel scheduling.

Angiogenesis contributes to solid-tumor growth and metastasis. ${ }^{9,10}$ Epithelial ovarian-cancer cell lines frequently express vascular endothelial growth factor (VEGF). ${ }^{11}$ Decreased VEGF expression is associated with reductions in tumor vascularization and angiogenesis and with prolonged survival. ${ }^{12}$ Bevacizumab (Avastin, Roche) is a monoclonal antibody that binds to all isoforms of the VEGF-receptor ligand VEGF-A, with evidence of efficacy in metastatic colorectal and lung cancers, as well as activity in renal, breast, and brain cancers. ${ }^{13-15}$ Phase 2 trials of bevacizumab in women with ovarian cancer have shown tumor responses and delayed disease progression. ${ }^{16-18}$

The Gynecologic Cancer InterGroup (GCIG) International Collaboration on Ovarian Neoplasms (ICON7) trial and the complementary Gynecologic Oncology Group study 0218 (GOG-0218) (ClinicalTrials.gov number, NCT00262847) were designed to investigate the addition of bevacizumab to standard chemotherapy in the first-line treatment of ovarian cancer. The results of the GOG-0218 study are reported elsewhere in this issue of the Journal by Burger et al. ${ }^{19}$

\section{METHODS}

STUDY DESIGN AND CONDUCT

ICON7 is led by the U.K. Medical Research Council Clinical Trials Unit (MRC CTU) and was designed by members of the Trial Management Group, who reviewed and approved the protocol. The Trial Management Group included represen- tatives from GCIG and F. Hoffmann-La Roche. An independent international trial steering committee and an independent data and safety monitoring committee provided oversight. Depending on national requirements, approval by ethics committees was obtained at each clinical site, nationally, or both. Data management was conducted by independent contract research organizations and MRC CTU (see the Supplementary Appendix, available with the full text of this article at NEJM.org). Data analysis and interpretation and preparation of the manuscript were performed independently by the funders and the sponsor (MRC). All authors had full access to all study data, had responsibility for the decision to submit the manuscript for publication, and vouch for the accuracy, integrity, and completeness of the data as reported. The trial was conducted in accordance with the protocol. (The protocol, including the statistical analysis plan, is available at NEJM.org.)

\section{ELIGIBILITY REQUIREMENTS}

After surgery, women were eligible for enrollment if they had histologically confirmed, highrisk, early-stage disease (International Federation of Gynecology and Obstetrics [FIGO] stage I or IIA and clear-call or grade 3 tumors) or advanced (FIGO stage IIB to IV) epithelial ovarian cancer, primary peritoneal cancer, or fallopian-tube cancer (based on local histopathological findings). Additional eligibility criteria were an Eastern Cooperative Oncology Group (ECOG) performance status of 0 to 2 (on a scale from 0 to 4 , with 0 indicating normal activity, 1 symptomatic but ambulatory self-care possible, 2 ambulatory more than $50 \%$ of the time, 3 ambulatory $50 \%$ of the time or less and nursing care required, and 4 bedridden and possibly requiring hospitalization) and adequate coagulation values and bone marrow, liver, and renal function, with no plans for further surgery before disease progression. All the patients provided written informed consent.

\section{RANDOMIZATION AND TREATMENTS}

Randomization was performed centrally by an interactive telephone or Web-based system, with stratification according to GCIG group, FIGO stage and residual disease (i.e., FIGO stages I to III and $\leq 1 \mathrm{~cm}$ of residual disease, stages I to III and $>1 \mathrm{~cm}$ of residual disease, or stage III [inoperable] or IV), and planned interval between surgery and initiation of chemotherapy ( $\leq 4$ weeks or $>4$ weeks). 
Enrollment of women with high-risk early-stage disease was restricted to $10 \%$ of the total study population.

Women were randomly assigned in a 1:1 ratio to receive carboplatin (area under the curve [AUC], 5 or 6) and paclitaxel (175 mg per square meter of body-surface area), given every 3 weeks for 6 cycles (standard-chemotherapy group), or to the same regimen plus bevacizumab (7.5 mg per kilogram of body weight), given concurrently every 3 weeks for 5 or 6 cycles and continued for 12 additional cycles or until disease progression (bevacizumab group). Bevacizumab was omitted at cycle 1 to avoid delayed wound healing if chemotherapy was started within 4 weeks of surgery. Cycles of bevacizumab that were omitted for any reason were not replaced.

\section{ASSESSMENTS}

Assessments were performed at the same time points in the two treatment groups. Clinical assessments and cancer antigen 125 (CA-125) measurements were performed before each cycle of chemotherapy, then every 6 weeks in year 1, every 3 months during years 2 and 3, every 6 months during years 4 and 5, and then yearly. After disease progression, follow-up assessments were performed every 6 months for the first 5 years, then yearly. Computed tomography (CT) or magnetic resonance imaging (MRI) was performed at baseline, after chemotherapy cycles 3 and 6 , at 9 and 12 months after randomization, every 6 months in years 2 and 3, and then as clinically indicated until disease progression. All tumor assessments were reviewed by the principal investigator, who was unaware of treatment assignments. Quality of life was assessed with the use of the European Organization for Research and Treatment of Cancer (EORTC) QLQ-C30 and QLQ-OV28 questionnaires (see the Supplementary Appendix).

\section{OUTCOME MEASURES}

The principal outcomes of interest were progression-free survival and overall survival. Other outcomes included biologic progression-free interval, response to therapy, toxicity, and quality of life.

Progression-free survival was calculated from the date of randomization to the date of the first indication of disease progression or death, whichever occurred first; the data for patients who were alive without disease progression were cen- sored as of the date of their last assessment. Sensitivity analyses were performed in which data from patients who were alive without disease progression were censored at the date of the last imaging assessment. Disease progression was defined according to the Response Evaluation Criteria in Solid Tumors (RECIST) ${ }^{20}$ guidelines (see the Supplementary Appendix) on the basis of radiologic, clinical, or symptomatic indicators of progression and did not include isolated asymptomatic progression on the basis of CA-125 levels. The biologic progression-free interval was calculated from the date of randomization to the date of the first CA-125-based progression ${ }^{21}$ or first RECIST-based progression, whichever occurred first (see the Supplementary Appendix). Overall survival was calculated from the date of randomization to the date of death from any cause; data for patients still alive were censored at the date the patient was last known to be alive. Best overall response was assessed in patients with measurable disease at baseline who received at least one cycle of per-protocol treatment and was defined as the best confirmed response recorded from the start of treatment until 70 days after the last dose of per-protocol treatment.

\section{STATISTICAL ANALYSIS}

The primary analysis was carried out with the use of an unstratified log-rank test for the difference in the distribution of progression-free survival between the two groups. Other planned analyses included a log-rank test that stratified for factors used for randomization (excluding GCIG groups to limit the number of categories being tested); Cox regression analyses that adjusted for baseline covariates to assess the robustness of the result if the proportional-hazards assumption held; flexible parametric survival models $^{22}$ to smooth survival curves and estimate survival differences with the use of all survival data collected; and interaction analyses to explore the difference in the relative size of treatment effects in subgroups classified according to baseline characteristics, high risk for progression (i.e., FIGO stage IV disease or FIGO stage III disease and $>1.0 \mathrm{~cm}$ of residual disease after debulking surgery), and stratification factors. All efficacy analyses for progression-free survival included all patients who had been randomly assigned to treatment (i.e., the intention-to-treat population). Safety analyses included patients who had 
received at least one cycle of the assigned protocol treatment. All reported P values are two-sided, and all statistical analyses were performed at MRC CTU. The magnitude and timing of treatment effect were explored with the use of hazard functions, including a formal test of the proportional-hazards assumption.

With an estimated median progression-free survival of 18 months and median overall survival of 43 months in the standard-chemotherapy group, the trial was designed to detect a $28 \%$ increase in median progression-free survival to 23 months (hazard ratio, 0.78) and a $23 \%$ increase in median overall survival to 53 months (hazard ratio, 0.81) in the bevacizumab group. At a $5 \%$ significance level and with $90 \%$ power for progression-free survival and $80 \%$ power for overall survival, a total of 684 progression-free survival events (i.e., disease progression or death) and 715 overall survival events were required. With 1520 women randomly assigned to treatment over a period of 2 years, the required progression-free survival and overall survival events were expected to occur 1 and 3 years, respectively, after entry of the last patient.

After submission of the primary analysis of the protocol-defined progression-free survival, regulatory authorities requested an overall survival analysis with at least 365 deaths $(50 \%$ of the required total number of deaths) and an update on progression-free survival with the use of the same data set.

\section{RESULTS}

\section{PATIENTS}

From December 2006 through February 2009, 1528 women were enrolled at 263 centers in the United Kingdom, Germany, France, Canada, Australia, New Zealand, Denmark, Finland, Norway, Sweden, and Spain. The cutoff date for the primary analysis was February 28, 2010, by which time a total of 759 progression-free survival events had been observed. The treatment groups were well balanced with respect to baseline characteristics. The median age was 57 years, and $94 \%$ of the patients had an ECOG performance status of 0 or $1 ; 90 \%$ had epithelial ovarian cancer; $9 \%$ had high-risk early-stage disease; $30 \%$ were at high risk for progression; $21 \%$ had FIGO stage III, IIIA, or IIIB disease; $70 \%$ had FIGO stage IIIC or IV disease; $69 \%$ had a serous histologic type; and
$26 \%$ had more than $1.0 \mathrm{~cm}$ of residual disease after surgical debulking (Table $1 S$ in the Supplementary Appendix).

\section{STUDY TREATMENTS RECEIVED}

More than $90 \%$ of the women in both groups received 6 cycles of chemotherapy $(91 \%$ in the standard-therapy group and $94 \%$ in the bevacizumab group). Women in the bevacizumab group who started chemotherapy at or before 4 weeks after surgery received a median of 16 cycles (interquartile range, 11 to 17) and those who started chemotherapy more than 4 weeks after surgery received a median of 17 cycles (interquartile range, 12 to 18$) ; 470$ patients (62\%) continued to receive bevacizumab through cycle 18, with 324 patients $(42 \%)$ missing no infusions. In the standardtherapy group, 1 patient received one dose of bevacizumab at cycle 1 in error. A total of 75 patients (5\%) (48 in the standard-therapy group and 27 in the bevacizumab group) received additional chemotherapy or bevacizumab before disease progression. After disease progression, a maximum of 44 patients (3\%) (30 in the standard-therapy group and 14 in the bevacizumab group) may have received further antiangiogenic treatment; the exact numbers are not known, since many patients are enrolled in blinded studies (Fig. 1).

\section{ADVERSE EVENTS}

Five deaths related to treatment or to treatment and disease were reported: one in the standardtherapy group (due to central nervous system ischemia) and four in the bevacizumab group (one each from gastrointestinal perforation, intracerebral hemorrhage, recurrent bowel perforation and ovarian cancer, and neutropenic sepsis and ovarian cancer). Adverse events of grade 3 or higher were reported in $56 \%$ of the women in the standard-therapy group and in $66 \%$ of the women in the bevacizumab group. Bevacizumab treatment appeared to be associated with an increase in bleeding (mainly grade 1 mucocutaneous bleeding), hypertension of grade 2 or higher ( $18 \%$ with bevacizumab vs. $2 \%$ with standard therapy), thromboembolic events of grade 3 or higher ( $7 \%$ with bevacizumab vs. $3 \%$ with standard therapy), and gastrointestinal perforations (occurring in 10 patients in the bevacizumab group vs. 3 patients in the standard-therapy group) (Table 1). In the bevacizumab group, the percentage of patients in whom abscesses, fistulas, or 


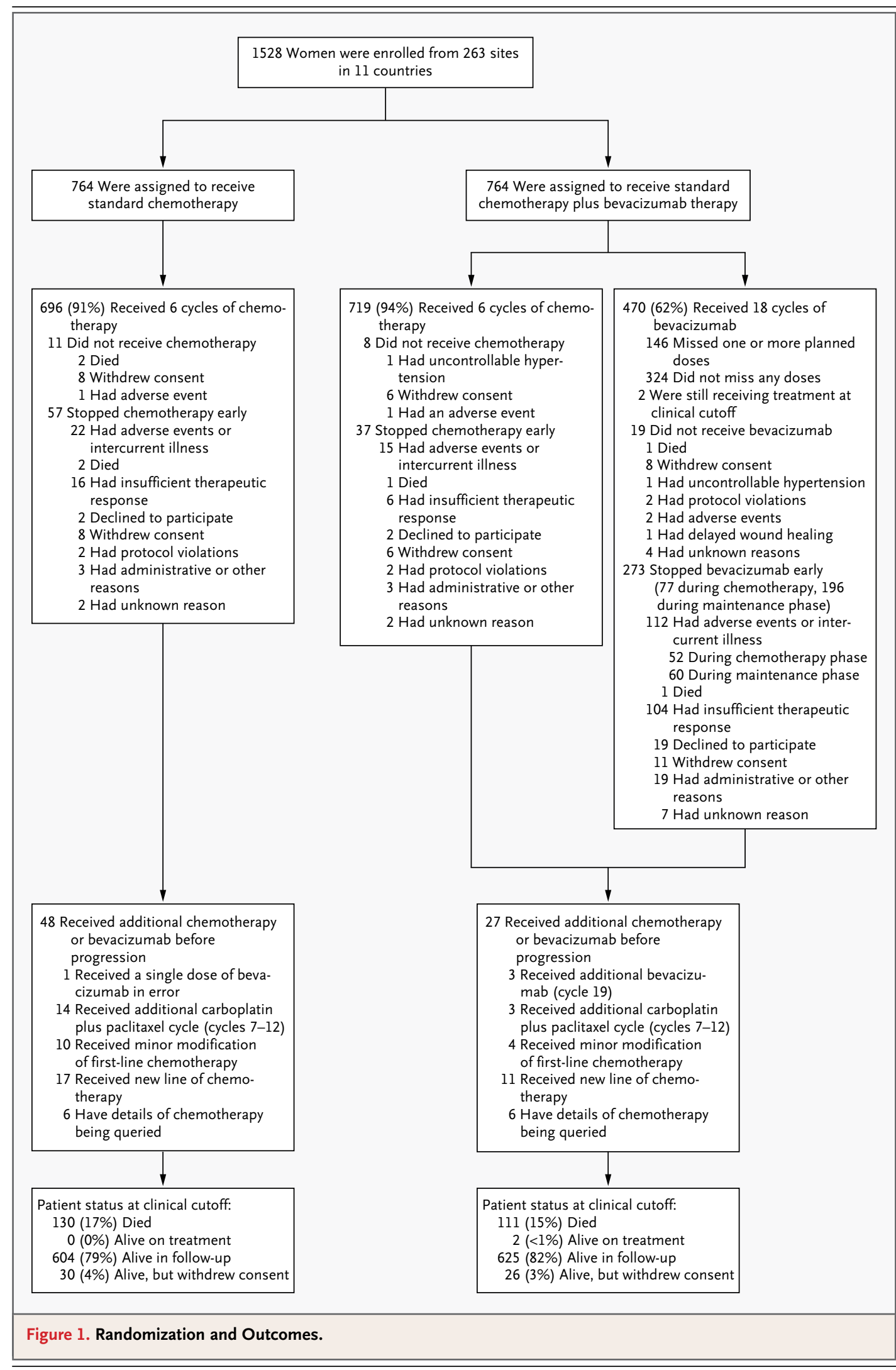


gastrointestinal perforations developed was similar in the group that received bevacizumab with their first cycle of chemotherapy and in the group that did not (3\% [19 of 611] and 4\% [6 of 135], respectively).

\section{BEST OVERALL RESPONSE}

The rate of complete or partial remission was $48 \%$ in the standard-therapy group and $67 \%$ in the bevacizumab group - a difference of 19 percentage points ( $95 \%$ confidence interval [CI], 11 to 28; $\mathrm{P}<0.001$ ) (Table $2 \mathrm{~S}$ in the Supplementary Appendix).

\section{PROGRESSION-FREE SURVIVAL}

With a median follow-up of 19.4 months, disease progression or death occurred in 759 patients (392 in the standard-therapy group and 367 in the bevacizumab group). The median progressionfree survival was 17.3 months in the standardtherapy group and 19.0 months in the bevacizumab group. A comparison of Kaplan-Meier curves for progression-free survival showed a significant difference between the two groups (estimated hazard ratio for progression or death in the bevacizumab group, $0.81 ; 95 \% \mathrm{CI}, 0.70$ to 0.94 ; $\mathrm{P}=0.004$ ) (Fig. 2A). There was clear evidence of nonproportional hazards (test of proportional hazards, $\mathrm{P}<0.001)$. The effect of bevacizumab changed over time (Fig. 2B) and was maximal at 12 months, with an improvement in progressionfree survival at this time of $15.1 \%$ (95\% CI, 10.7 to 19.5) as compared with standard therapy; thereafter, the effect was diminished so that progression-free survival was slightly higher in the standard-therapy group at 24 months. In detecting nonproportional hazards, the log-rank test remains useful, but the conventional hazard ratio is not meaningful; a better estimate of treatment effect is provided by the restricted mean difference (the difference in areas under the whole length of the progression-free survival curves). The restricted mean values for progression-free survival in the standard-therapy and bevacizumab groups, estimated with the use of all data obtained up to 36 months after randomization, were 20.3 months and 21.8 months, respectively - a difference of 1.5 months ( $95 \%$ CI, 0.1 to 2.9). Sensitivity analyses censoring data obtained at the last radiologic tumor assessment led to similar conclusions (Fig. 1S, Panel a, in the Supplementary Appendix) (hazard ratio for progression or death in the bevacizumab group, $0.79 ; 95 \% \mathrm{CI}, 0.68$ to
$0.91 ; \mathrm{P}=0.001$ by the log-rank test [nonproportional hazards detected]; restricted mean difference, 1.2 months [progression-free survival of 17.8 months with standard therapy vs. 19.0 months with bevacizumab], estimated at 30 months after randomization).

Among patients at high risk for progression (Fig. 2C), the estimated median progression-free survival was 10.5 months with standard therapy, as compared with 15.9 months with bevacizumab (hazard ratio for progression or death in the bevacizumab group, 0.68 ; $95 \% \mathrm{CI}, 0.55$ to 0.85 ; $\mathrm{P}<0.001)$. The restricted mean values at 36 months were 13.1 months with standard therapy and 16.5 months with bevacizumab.

Isolated CA-125-based progression occurred in 254 women (117 in the standard-therapy group and 137 in the bevacizumab group). Disease progression as assessed on the basis of CA-125, RECIST, or both occurred in 767 women (395 in the standardtherapy group and 372 in the bevacizumab group). The hazard ratio for biologic progression-free survival with bevacizumab, as compared with standard therapy, was 0.83 (95\% CI, 0.72 to 0.96 ; $\mathrm{P}=0.009$ ) (Fig. 1S, Panel b, in the Supplementary Appendix).

Exploratory analyses of potential interactions are summarized in Figure 3, and in Figure 3S in the Supplementary Appendix. There is a suggestion of a greater benefit with bevacizumab in women with a worse ECOG performance score than for those with a normal performance score $(\mathrm{P}=0.02$ for interaction).

\section{OVERALL SURVIVAL AND QUALITY OF LIFE}

Overall survival data are not final; the final results are due in 2013 (Fig. 2D). There were 241 deaths: 231 (96\%) were disease-related (128 in the standardtherapy group and 103 in the bevacizumab group), 5 were treatment-related, and 5 were due to other causes (see the Supplementary Appendix). Both groups showed improvement in global quality of life over time; although differences between the two groups were consistently present, these were small and not considered to be clinically significant (i.e., there was less than a 10-point difference) (Fig. $3 S$ and Table $3 S$ in the Supplementary Appendix).

\section{UPDATED ANALYSES}

The cutoff date for updated analyses of progressionfree survival and overall survival was November 30, 2010, after a median follow-up period of 28 months 


\section{Table 1. Summary of Adverse Events."}

\section{Adverse Events}

Any event

None

Grade 1 or 2

Grade 3 or 4

Grade 5

Any bleeding

None

Grade $\geq 3$

Bleeding other than mucocutaneous, tumor-associated, or within CNS

None

Grade 1 or 2

Grade $\geq 3$

Mucocutaneous bleeding

None

Grade 1 or 2

Grade $\geq 3$

Tumor-associated bleeding

Bleeding within CNS

None

Grade 1 or 2

Grade $\geq 3$

Abscess and fistula

None

Grade 1 or 2

Grade $\geq 3$

Gastrointestinal perforation

None

Grade 1 or 2

Grade $\geq 3$

Hypertension

None

Grade 1

Grade 2

Grade $\geq 3$

Proteinuria

None

Grade 1 or 2

Grade $\geq 3$
Standard Chemotherapy

( $N=753)$

number of patients (percent)
Bevacizumab

$(\mathrm{N}=745)$

$3(<1)$

331 (44)

0

$410(54)$

254 (34)

9 (1)

8 (1)

$666(88)$

$450(60)$

85 (11)

$286(38)$

$2(<1)$

9 (1)

712 (95)

688 (92)

39 (5)

55 (7)

$2(<1)$

$2(<1)$

$698(93)$

469 (63)

55 (7)

$271(36)$

0

5 (1)

0

0

$753(100)$

743 (99)

0

0

0

$2(<1)$

743 (99)

732 (98)

$3(<1)$

$7(1)$

7 (1)

$6(1)$

750 (99)

735 (99)

0

0

$3(<1)$

$10(1)$

706 (94)

$552(74)$

31 (4)

57 (8)

14 (2)

90 (12)

$2(<1)$

$46(6)$

734 (97)

712 (96)

18 (2)

29 (4)

$1(<1)$

The New England Journal of Medicine 


\begin{tabular}{|c|c|c|}
\hline \multirow[t]{2}{*}{ Adverse Events } & $\begin{array}{l}\text { Standard Chemotherapy } \\
\qquad(N=753)\end{array}$ & $\begin{array}{c}\text { Bevacizumab } \\
(\mathrm{N}=745)\end{array}$ \\
\hline & \multicolumn{2}{|c|}{ number of patients (percent) } \\
\hline \multicolumn{3}{|l|}{ Any thromboembolic event } \\
\hline None & $708(94)$ & $665(89)$ \\
\hline Grade 1 or 2 & $22(3)$ & $29(4)$ \\
\hline Grade $\geq 3$ & $23(3)$ & $51(7)$ \\
\hline \multicolumn{3}{|l|}{ Venous thromboembolic event } \\
\hline None & $722(96)$ & $695(93)$ \\
\hline Grade 1 or 2 & $18(2)$ & $18(2)$ \\
\hline Grade $\geq 3$ & $13(2)$ & $32(4)$ \\
\hline \multicolumn{3}{|l|}{ Arterial thromboembolic event } \\
\hline None & 742 (99) & $718(96)$ \\
\hline Grade 1 or 2 & $1(<1)$ & $7(1)$ \\
\hline Grade $\geq 3$ & $10(1)$ & $20(3)$ \\
\hline \multicolumn{3}{|l|}{ Local thrombosis } \\
\hline None & 750 (99) & 740 (99) \\
\hline Grade 1 or 2 & $3(<1)$ & $5(1)$ \\
\hline Grade $\geq 3$ & 0 & 0 \\
\hline \multicolumn{3}{|l|}{ Neutropenia } \\
\hline None & $534(71)$ & $534(72)$ \\
\hline Grade 1 or 2 & $105(14)$ & $88(12)$ \\
\hline Grade $\geq 3$ & $114(15)$ & $123(17)$ \\
\hline \multicolumn{3}{|l|}{ Febrile neutropenia } \\
\hline None & $738(98)$ & $724(97)$ \\
\hline Grade 1 or 2 & $1(<1)$ & $2(<1)$ \\
\hline Grade $\geq 3$ & $14(2)$ & $19(3)$ \\
\hline \multicolumn{3}{|l|}{ Thrombocytopenia } \\
\hline None & $684(91)$ & $652(88)$ \\
\hline Grade 1 or 2 & $54(7)$ & $67(9)$ \\
\hline Grade $\geq 3$ & $15(2)$ & $26(3)$ \\
\hline Reversible posterior leukoencephalopathy syndrome & 0 & 0 \\
\hline \multicolumn{3}{|l|}{ Congestive heart failure } \\
\hline None & $750(99)$ & $742(99)$ \\
\hline Grade 1 or 2 & 0 & $1(<1)$ \\
\hline Grade $\geq 3$ & $3(<1)$ & $2(<1)$ \\
\hline \multicolumn{3}{|l|}{ Complication of wound healing } \\
\hline None & $737(98)$ & $708(95)$ \\
\hline Grade 1 or 2 & $13(2)$ & $27(4)$ \\
\hline Grade $\geq 3$ & $3(<1)$ & $10(1)$ \\
\hline \multicolumn{3}{|l|}{ Hyperbilirubinemia } \\
\hline None & $753(100)$ & 742 (99) \\
\hline Grade 1 or 2 & 0 & $2(<1)$ \\
\hline Grade $\geq 3$ & 0 & 0 \\
\hline
\end{tabular}

* CNS denotes central nervous system. 


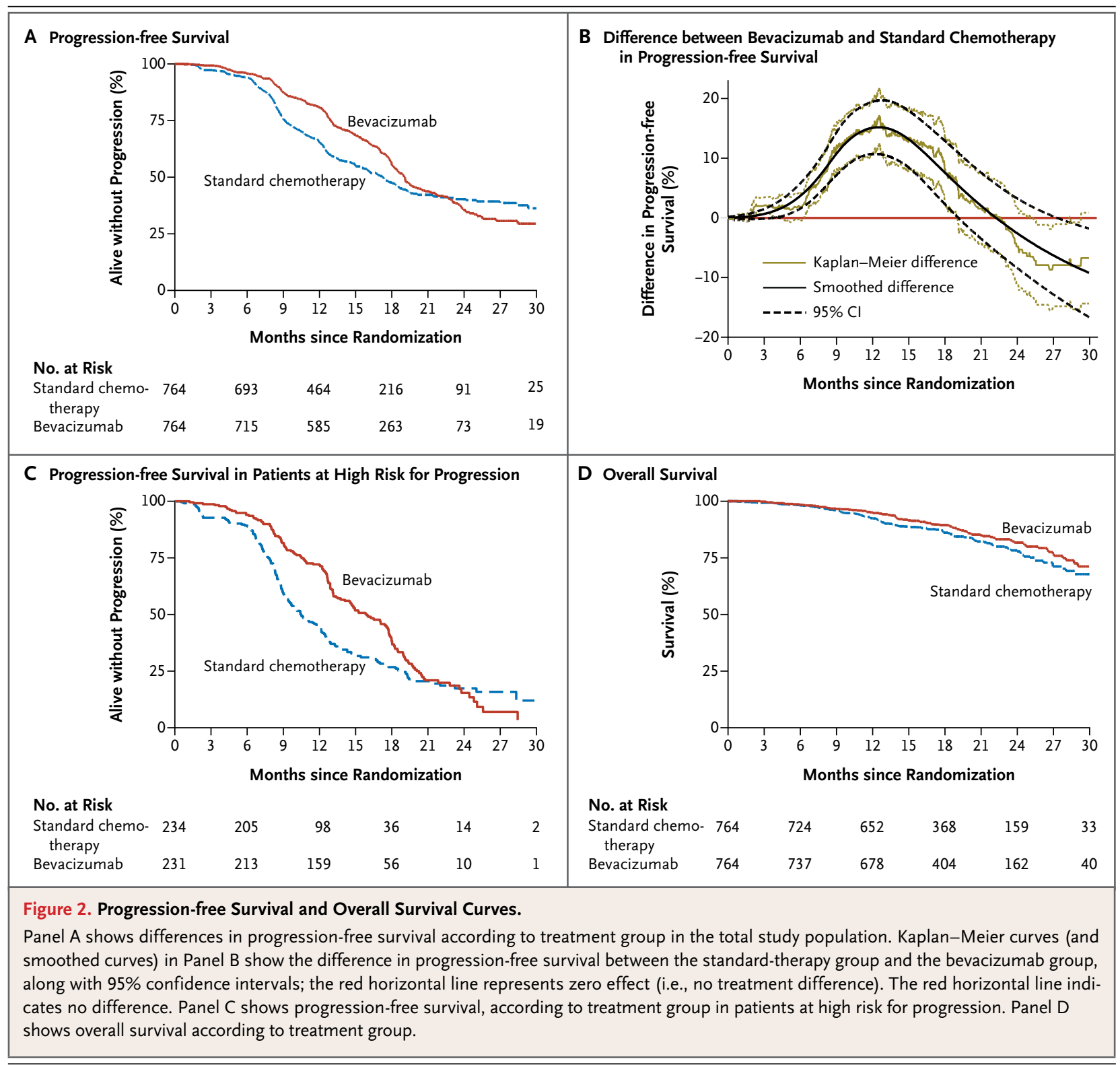

and a total of 378 deaths observed. Table 2 shows the results of the primary and updated analyses for progression-free survival and overall survival.

\section{Progression-free Survival}

A total of 934 progression-free survival events (progression or death) were reported (464 in the standard-therapy group and 470 in the bevacizumab group). Results were very similar to those of the primary analysis, again showing clear evidence of a nonproportional hazard $(\mathrm{P}=0.001)$ (Table 2, and Fig. 5S, Panel a, in the Supplementary Appendix). A long-term improvement in progression-free survival was seen with bevacizumab, as compared with standard therapy, with median durations of 19.8 months in the bevacizumab group and 17.4 months in the standard-therapy group, and corresponding restricted means of 24.1 months and 22.4 months at 42 months (hazard ratio for progression or death in the bevacizumab group, $0.87 ; 95 \% \mathrm{CI}, 0.77$ to $0.99 ; \mathrm{P}=0.04$ ).

The test for interaction suggests that the size of the effect of bevacizumab differed between patients at high risk for progression and the rest of the study population $(\mathrm{P}=0.06)$ (Fig. $4 \mathrm{~S}$, Panels a and b, in the Supplementary Appendix). Among the 465 women at high-risk for progression, 386 (83\%) had disease progression or 


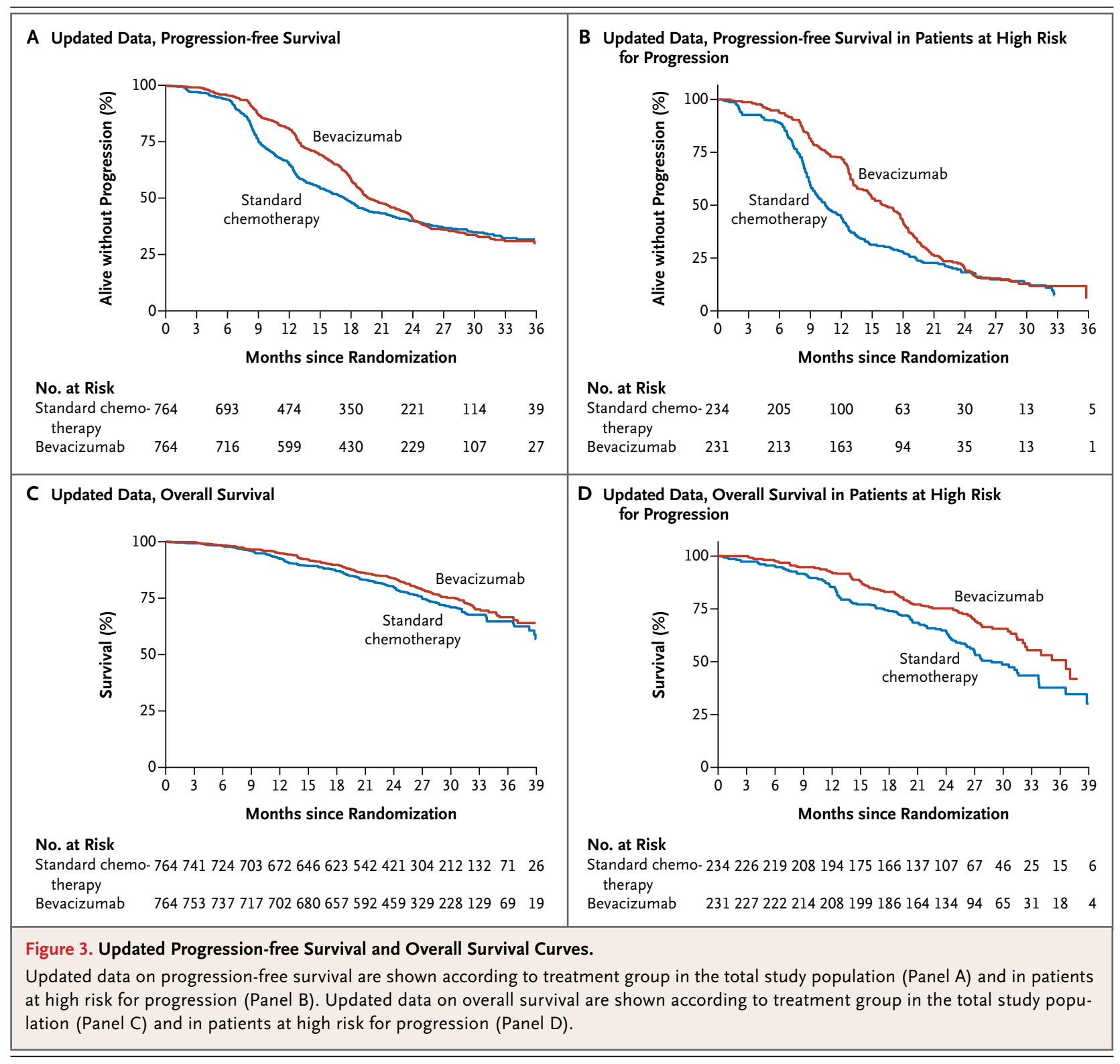

died, and the benefit with respect to progressionfree survival was greater with bevacizumab than with standard therapy, with median durations of 10.5 months in the standard-therapy group and 16.0 months in the bevacizumab group and restricted means of 14.5 months and 18.1 months at 42 months in the two groups, respectively (hazard ratio for progression or death in the bevacizumab group, 0.73 ; $95 \% \mathrm{CI}, 0.60$ to 0.93 ; $\mathrm{P}=0.002$ ). The updated progression-free survival curves were similar to those obtained in the primary analyses up to 24 months of follow-up (Fig. 3B).

\section{Overall Survival}

A total of 146 new deaths (all disease-related) were reported, making the total number of deaths 378 (200 in the standard-therapy group and 178 in the bevacizumab group). In contrast to the data for progression-free survival, there was no evidence of nonproportional hazards. A comparison of the Kaplan-Meier curves resulted in a hazard ratio for death in the bevacizumab group of 0.85 (95\% CI, 0.69 to $1.04 ; \mathrm{P}=0.11$ ). The early indication of a trend toward improved overall survival with bevacizumab has not changed (Fig. 3C).

The test for interaction suggests that the size 
Table 2. Results of Primary and Updated Analyses of Progression-free Survival and Overall Survival with Standard Chemotherapy Alone versus Standard Therapy plus Bevacizumab.*

Variable Primary Analysis

\section{Updated Analysis}

Standard

Progression-free survival

Bevacizumab

Standard

Chemotherapy

Bevacizumab

All patients

No. of events

Median (mo)

392

367

19.0

464

470

Hazard ratio $(95 \% \mathrm{Cl})$

17.3

$0.81(0.70-0.94)$

17.4

19.8

$P$ value $\dagger$

0.004

$0.87(0.77-0.99)$

$<0.001$

0.04

$P$ valuet:

Restricted mean (mo)

At 36 mo

20.3

21.8

NA

20.6

22.5

At $42 \mathrm{mo}$

NA

173

196

190

No. of progression-free survival events

158

15.9

10.5

16.0

Hazard ratio $(95 \% \mathrm{Cl})$

10.5

$0.68(0.55-0.85)$

$<0.001$

$0.73(0.60-0.93)$

$<0.001$

0.002

$P$ valuet:

Restricted mean (mo)
At $36 \mathrm{mo}$

At $42 \mathrm{mo}$

Overall survivalq

All patients

No. of events

Median (mo)

1-year survival, per Kaplan-Meier estimates (\%)

Hazard ratio $(95 \% \mathrm{Cl})$

$P$ value'

Patients at high risk for progression』

No. of events

Median (mo)

Hazard ratio $(95 \% \mathrm{Cl})$

P value $†$
130

Not yet reached

93

13.1

NA
16.5

NA
111

Not yet reached

95

$0.81(0.63-1.04)$

0.098

No analyses performed
No analyses performed
$<0.001$
17.6

14.1

18.1 
of the effect of bevacizumab on overall survival differs between the patients at high risk for progression and the rest of the women studied $(\mathrm{P}=0.011)$ (Fig. 4S, Panels $\mathrm{c}$ and $\mathrm{d}$, in the Supplementary Appendix). Among the women at high risk for progression, there were 188 deaths (109 in the standard-therapy group and 79 in the bevacizumab group) (Fig. 3D, and Fig. 4S, Panel d, in the Supplementary Appendix). The median overall survival is 28.8 months in the standard-therapy group and 36.6 months in the bevacizumab group, (hazard ratio for death in the bevacizumab group, $0.64 ; 95 \% \mathrm{CI}, 0.48$ to $0.85 ; \mathrm{P}=0.002$ ).

\section{DISCUSSION}

The use of bevacizumab (7.5 mg per kilogram) given concurrently with 5 or 6 cycles of platinumbased chemotherapy and continued for an additional 12 cycles improved progression-free survival by about 2 months and increased the response rate by $20 \%$. These data provide clear evidence of the biologic activity of bevacizumab, with a maximum improvement in progression-free survival of approximately $15 \%$ at 12 months, which disappeared by 24 months. The restricted mean difference summarized the treatment effect for the nonproportional hazards detected and showed an improvement in the mean progression-free survival of 1.5 to 2 months with bevacizumab. The progression-free survival and overall survival benefits were much greater among the patients at high risk for progression (improvements of 3.6 months [restricted mean] and 7.8 months [median], respectively) than among patients at lower risk. Both the timing of the maximal treatment effect, which coincided with the end of planned bevacizumab treatment (and was achieved in more than $70 \%$ of the women), and the larger treatment effect observed in women with more advanced disease, who were more likely to receive bevacizumab up to the time of disease progression, are intriguing and raise the possibility that prolonged therapy beyond 12 months, perhaps until disease progression, might further improve the outcome.

Treatment with bevacizumab did not affect the delivery of chemotherapy. However, bevacizumab did expand the range of toxic effects, including hypertension and bowel perforation.

As compared with the GOG-0218 study, the ICON7 study enrolled patients with advancedstage cancer with no visible residual disease, as well as some with high-risk early-stage disease; in the ICON7 study, half the dose of bevacizumab was used (7.5 mg per kilogram, vs. $15 \mathrm{mg}$ per kilogram in the GOG-0218 study) for a shorter maintenance period (12 cycles, vs. 16 cycles in the GOG-0218 study). The dose of bevacizumab used in the ICON7 study is one of the licensed doses for metastatic colorectal cancer but is half the licensed dose for metastatic breast cancer. The prognosis for patients at high risk for progression in the ICON7 study was similar to that for patients in the GOG-0218 study, with median progression-free survival of 10.5 months in the standard-therapy group. A 3.6-month (restricted mean) improvement in progression-free survival was observed with bevacizumab, similar to that seen in the GOG-0218 study. ${ }^{19}$

For the whole ICON7 patient population, the overall magnitude of the benefit with respect to progression-free survival was relatively modest after the addition of bevacizumab to platinum-based chemotherapy and maintenance bevacizumab for the 12-cycle extension after chemotherapy was completed. Some will argue that final overall survival data are needed before the results can be fully interpreted. The apparently greater effect of bevacizumab in patients with a poor prognosis is encouraging. Final results for overall survival rates should be available in 2013 .

Supported by Roche and the National Institute for Health Research, through the National Cancer Research Network.

Disclosure forms provided by the authors are available with the full text of this article at NEJM.org.

We thank the women who participated in the trial and their families, Cancer Research U.K. (C2113/A6865) for peer review of the ICON7 trial proposal, the participating Gynecologic Cancer InterGroup groups (Arbeitsgemeinschaft Gynaekologische Onkologie Studiengruppe Ovarialkarzinom [AGO-OVAR], Australia New Zealand Gynaecological Oncology Group [ANZGOG], Grupo Español de Investigaciõn en Cancer de Ovario [GEICO], Association de Recherche sur les Cancers dont GynécologiquesGroupe d'Investigateurs Nationaux pour l'Etude des Cancers Ovariens et du Sein [ARCAGY-GINECO], Medical Research CouncilNational Cancer Research Institute [MRC-NCRI], Nordic Society for Gynecologic Oncology [NSGO], and NCIC Clinical Trials Group [NCICCTG]), and the 263 clinical sites and their staff. 


\section{APPENDIX}

The authors' affiliations are as follows: From St. James's Institute of Oncology, St. James's University Hospital (T.J.P., F.C.), and Leeds Institute of Molecular Medicine (D.S.), Leeds; St. Luke's Cancer Center, Royal Surrey County National Health Service (NHS) Foundation Trust, Guildford (S.E.); and Cambridge Cancer Trials Center, Cambridge University Hospitals NHS Foundation Trust, Cambridge (W.Q.) — all in the United Kingdom; the Medical Research Council Clinical Trials Unit (A.M.S., W.Q., M.K.B.P.) and University College London Biomedical Research Centre, University College London Hospitals (J.A.L.) — both in London; Städtischen Klinikum Solingen, Klinik für Gynäkologie und Geburtshilfe, Solingen (J.P.), Universitätsklinikum Ulm, Ulm (C.K.), Dr. Horst Schmidt Klinik, Wiesbaden (A.B.); Universitätsklinikum Essen, Essen (R.K.); and St. Vincentius Kliniken, Karlsruhe (A.S.) - all in Germany; Université Paris Descartes, Assistance Publique-Hôpitaux de Paris, Hôpitaux Universitaires Paris Centre, Paris (E.P.-L.); the Department of Gynecologic Oncology and Institute of Medical Informatics, Oslo University Hospital, Oslo (G.K.); the Department of Obstetrics and Gynecology, University of British Columbia, Vancouver (M.S.C.), and the Department of Obstetrics and Gynecology, Laval University, Quebec, QC (M.P.) — both in Canada; Australia New Zealand Gynaecological Oncology Group, Camperdown, NSW, Australia (P.B.); the Department of Hematology and Medical Oncology, Institute of Health Research Hospital Clinico, University of Valencia, Valencia, Spain (A.C.); Charité Campus Virchow-Klinikum, Berlin (J.S.); the University of Edinburgh Cancer Research Centre, Institute of Genetics and Molecular Medicine, Edinburgh (C.G.); Centre Catherine de Sienne, Nantes (A. Lortholary); and Service d'Oncologie Medicale, Assistance Publique-Hôpitaux de Paris, Hôpital Tenon, Paris (F.S.) - both in France; the Department of Oncology, Rigshospitalet, Copenhagen (M.R.M.); the Women's Clinic, Helsinki University Central Hospital, Helsinki (A. Leminen); and the Princess Margaret Hospital, Toronto (A.M.O.).

\section{REFERENCES}

1. Bookman MA, Brady MF, McGuire WP, et al. Evaluation of new platinum-based treatment regimens in advanced-stage ovarian cancer: a Phase III Trial of the Gynecologic Cancer Intergroup. J Clin Oncol 2009; 27:1419-25. [Erratum, J Clin Oncol 2009;27: 2305.]

2. du Bois A, Weber B, Rochon J, et al. Addition of epirubicin as a third drug to carboplatin-paclitaxel in first-line treatment of advanced ovarian cancer: a prospectively randomized gynecologic cancer intergroup trial by the Arbeitsgemeinschaft Gynaekologische Onkologie Ovarian Cancer Study Group and the Groupe d'Investigateurs Nationaux pour l'Etude des Cancers Ovariens. J Clin Oncol 2006;24:1127-35.

3. Pfisterer J, Weber B, Reuss A, et al. Randomized phase III trial of topotecan following carboplatin and paclitaxel in first-line treatment of advanced ovarian cancer: a gynecologic cancer intergroup trial of the AGO-OVAR and GINECO. J Natl Cancer Inst 2006;98:1036-45.

4. Hoskins P, Vergote I, Cervantes A, et al. Advanced ovarian cancer: phase III randomized study of sequential cisplatin-topotecan and carboplatin-paclitaxel vs. carboplatinpaclitaxel. J Natl Cancer Inst 2010;102: 1547-56.

5. Armstrong DK, Bundy B, Wenzel L, et al. Intraperitoneal cisplatin and paclitaxel in ovarian cancer. N Engl J Med 2006; 354:34-43.

6. Alberts DS, Liu PY, Hannigan EV, et al. Intraperitoneal cisplatin plus intravenous cyclophosphamide versus intravenous cisplatin plus intravenous cyclophosphamide for stage III ovarian cancer. N Engl J Med 1996;335:1950-5.

7. Markman M, Bundy BN, Alberts DS, et al. Phase III trial of standard-dose intravenous cisplatin plus paclitaxel versus moderately high-dose carboplatin followed by intravenous paclitaxel and intraperitoneal cisplatin in small-volume stage III ovarian carcinoma: an intergroup study of the Gynecologic Oncology Group, Southwestern Oncology Group, and Eastern Cooperative Oncology Group. J Clin Oncol 2001;19:1001 7.

8. Katsumata N, Yasuda M, Takahashi F, et al. Dose-dense paclitaxel once a week in combination with carboplatin every 3 weeks for advanced ovarian cancer: a phase 3, open-label, randomised controlled trial. Lancet 2009;374:1331-8.

9. Folkman J. Anti-angiogenesis: new concept for therapy of solid tumors. Ann Surg 1972;175:409-16.

10. Idem. What is the evidence that tumors are angiogenesis dependent? J Nat Cancer Inst 1990;82:4-6.

11. Yoneda J, Kuniyasu H, Crispens MA Price JE, Bucana CD, Fidler IJ. Expression of angiogenesis-related genes and progression of human ovarian carcinomas in nude mice. J Natl Cancer Inst 1998;90:447-54.

12. Huang S, Robinson JB, Deguzman A Bucana CD, Fidler IJ. Blockade of nuclear factor-kappaB signaling inhibits angiogenesis and tumorigenicity of human ovarian cancer cells by suppressing expression of vascular endothelial growth factor and interleukin 8. Cancer Res 2000; 60:5334-9.

13. Hurwitz H, Fehrenbacher L, Novotny W, et al. Bevacizumab plus irinotecan, fluorouracil, and leucovorin for metastatic colorectal cancer. N Engl J Med 2004;350:233542.

14. Sandler A, Gray R, Brahmer J. Randomised phase II/III trial of paclitaxel (P) plus carboplatin (C) with or without bevacizumab (NSC \#704865) in patients with advanced non-squamous non-small cell lung cancer (NSCLC): an Eastern Cooperative Oncology Group (ECOG) trial. Presented at the 41st Annual Meeting of the American Society of Clinical Oncology, Orlando, FL, May 13-17, 2005. abstract.

15. Eskens FA, Sleijfer S. The use of beva- cizumab in colorectal, lung, breast, renal and ovarian cancer: where does it fit? Eur J Cancer 2008;44:2350-6.

16. Burger RA, Sill MW, Monk BJ, Greer BE, Sorosky JI. Phase II trial of bevacizumab in persistent or recurrent epithelial ovarian cancer or primary peritoneal cancer: a Gynecologic Oncology Group study. J Clin Oncol 2007;25:5165-71.

17. Cannistra SA, Matulonis UA, Penson RT, et al. Phase II study of bevacizumab in patients with platinum-resistant ovarian cancer or peritoneal serous cancer. J Clin Oncol 2007;25:5180-6. [Erratum, J Clin Oncol 2008;26:1773.]

18. Garcia AA, Hirte H, Fleming G, et al. Phase II clinical trial of bevacizumab and low-dose metronomic oral cyclophosphamide in recurrent ovarian cancer: a trial of the California, Chicago, and Princess Margaret Hospital Phase II Consortia. J Clin Oncol 2008;26:76-82.

19. Burger RA, Brady MF, Bookman MA, et al. Incorporation of bevacizumab in the primary treatment of ovarian cancer. $\mathrm{N}$ Engl J Med 2011;365:2473-83.

20. Therasse P, Arbuck SG, Eisenhauer EA, et al. New guidelines to evaluate the response to treatment in solid tumors: European Organization for Research and Treatment of Cancer, National Cancer Institute of the United States, National Cancer Institute of Canada. J Natl Cancer Inst 2000;92:205-16.

21. Rustin GJ, Quinn M, Thigpen T, et al. Re: new guidelines to evaluate the response to treatment in solid tumors (ovarian cancer). J Natl Cancer Inst 2004;96:487-8.

22. Royston P, Parmar MKB. Flexible proportional-hazards and proportional-odds models for censored survival data, with application to prognostic modelling and estimation of treatment effects. Stat Med 2002;21:2175-97.

Copyright (C) 2011 Massachusetts Medical Society. 